\title{
46. On the Cauchy Problem for Weakly Hyperbolic Systems
}

By Hideo YamaHaRA

Department of Mathematics, Kyoto University

(Comm. by Kôsaku YosidA, M. J. A., April 12, 1976)

$\S 1$. Introduction. In this paper we consider the $\varepsilon$-well-posedness for the Cauchy problem of the first order system:

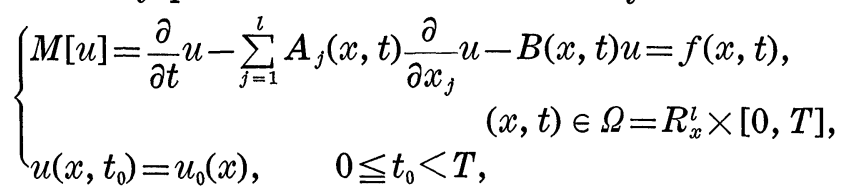

where $A_{j}(x, t)$ and $B(x, t)$ are $(m, m)$ matrices whose elements belong to the class $\mathcal{B}(\Omega)$ (in the sense of L. Schwartz [5]).

We suppose that $A(x, t, \xi)=\sum_{j=1}^{l} A_{j}(x, t) \xi_{j}$ is not diagonalizable. Such a case has been treated by V. M. Petkov with the method of asymptotic expansions ([6], [7]).

Here we shall approach this problem in a different viewpoint from his and propose a more concrete condition which is necessary and sufficient for the $\mathcal{E}$-well-posedness of (1.1). Our proof is much due to, socalled, the method of energy estimates (see S. Mizohata [2], S. Mizohata and Y. Ohya [3], [4]). The forthcoming paper will give the detailed proofs.

§ 2. Levi's condition and an energy estimate. As indicated in $\S 1$, throughout this paper we assume the following:

(2.1) The multiplicities of the characteristic roots are constant and at most double, more precisely,

$$
\operatorname{det}(\tau I-A(x, t ; \xi))=\prod_{i=1}^{s}\left(\tau-\lambda_{i}(x, t ; \xi)\right)^{2} \quad \prod_{j=s+1}^{m-s}\left(\tau-\lambda_{j}(x, t ; \xi)\right) .
$$

(2.2) The roots $\lambda_{i}(x, t ; \xi)$ are real and distinct for $(x, t ; \xi) \in \Omega$ $\times\left(R_{\xi}^{\imath} \backslash\{0\}\right),(i=1,2, \cdots, m-s)$.

(2.3) For $i=1,2, \cdots, s, \operatorname{rank}\left(\lambda_{i}(x, t ; \xi) I-A(x, t ; \xi)\right)=m-1$, independently of $(x, t ; \xi)$.

Proposition 2.1. Suppose (2.1) and (2.3), then there exists a $(m, m)$ matrix $N(x, t ; \xi)$ which satisfies

(i ) $N(x, t ; \xi) A(x, t ; \xi)=D(x, t ; \xi) N(x, t ; \xi)$, where 


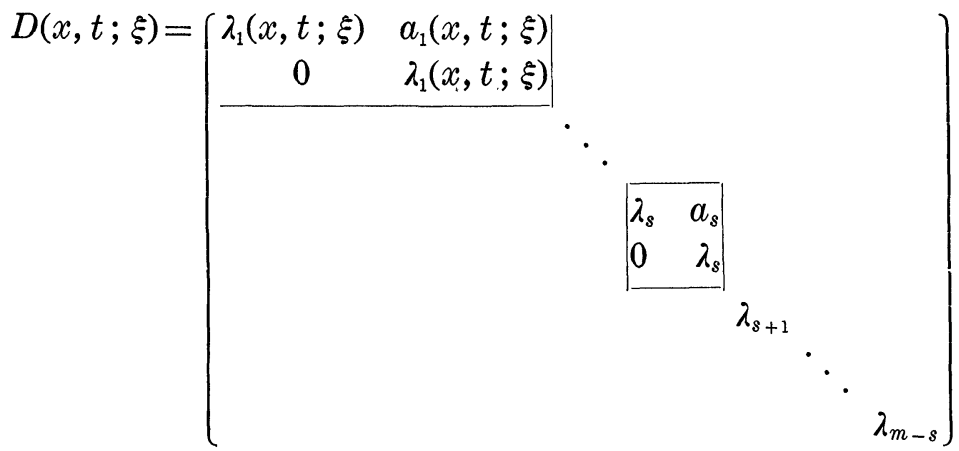

and $a_{i}(x, t ; \xi)$ are homogeneous of degree 1 in $\xi,(i=1,2, \cdots, s)$.

(ii) $N(x, t ; \xi)$ is homogeneous of degree 0 in $\xi$.

(iii) $|\operatorname{det} N(x, t ; \xi)| \geqq \delta(>0)$ for $(x, t ; \xi) \in \Omega \times\left(R_{\xi}^{l} \backslash\{0\}\right)$.

Now we consider the equations :

$$
M[u]=\left[\frac{\partial}{\partial t}-i \mathcal{A}(x, t ; D)-B(x, t)\right] u(x, t)=f(x, t) .
$$

Operate the pseudo-differential operator (hereafter we write this p.d.op.) $\mathscr{l}(x, t ; D)$ with the symbol $N(x, t ; \xi)$, then we have

$$
\left[\frac{\partial}{\partial t}-i \mathscr{D}(x, t ; D)-\mathscr{B}_{1}(x, t ; D)\right](\mathscr{N} u)=\mathscr{N} f+\mathcal{C}(x, t ; D) u,
$$

where $\sigma\left(\mathscr{D}(x, t ; D)=D(x, t ; \xi), \mathscr{B}_{1}(x, t ; D)\right.$ is a p.d.o.p., homogeneous of order 0 and $\mathcal{C}(x, t ; D)$ is of order -1 .

Denote the symbol of $\mathscr{B}_{1}(x, t ; D)$ by $B_{1}(x, t ; \xi)$, and its $(i, j)$-entry by $b_{i, j}^{(1)}(x, t ; \xi)$. Let us introduce the following condition:

(C.A) All the symbols $b_{2 i, 2 i-1}^{(1)}(x, t ; \xi)$ are identically zero for $(x, t ; \xi) \in \Omega \times R_{\xi}^{l},(i=1,2, \cdots, s)$.

Theorem 2.1. Suppose the condition (C.A) and let $u(t)=u(x, t)$ be the solution of (1.1) belonging to $\mathcal{E}_{t}^{1}\left(\mathscr{D}_{L^{2}}^{k}\right)$, then there exists a constant $C$, such that

$$
\|u(t)\|_{k-1} \leqq C\left[\left\|u\left(t_{0}\right)\right\|_{k}+\int_{t_{0}}^{t}\|f(s)\|_{k} d s\right],
$$

for any integer $k \geqq 2$.

We show the outline of this proof. First we introduce the operator $g(D)$ defined by $g(D)^{t}\left(u_{1}, \cdots, u_{n}\right)={ }^{t}\left((1+\Lambda)^{-1} u_{1}, u_{2},(1+\Lambda)^{-1} u_{3}, u_{4}, \cdots\right.$, $\left.(1+\Lambda)^{-1} u_{2 s-1}, u_{2 s}, u_{2 s+1}, \cdots, u_{m}\right)$. Then we have

$$
\left[\frac{\partial}{\partial t}-i \mathscr{D}_{1}(x, t ; D)-\mathscr{B}_{2}(x, t ; D)\right](g \mathscr{T} u)=g \Re_{f}+g \mathcal{C} u,
$$

where $\mathscr{D}_{1}(x, t ; D)$ is homogeneous of order 1 and diagonalizable, and $\mathcal{B}_{2}(x, t ; D)$ is of order 0 . Now by an almost procedure we can prove Theorem 2.1.

§3. Condition (C.A) and the influence domain. We shall represent the condition (C.A) more explicitly. For this purpose we 
calculate the symbol of $\mathscr{B}_{1}(x, t ; D)$.

$\sigma\left(\mathcal{B}_{1}(x, t ; D)\right)=$ principal symbol of $\left[i(\mathscr{A} \mathcal{A}-\mathscr{D} \mathscr{N})-\mathfrak{N}_{t}^{\prime}+\mathscr{I} B\right] \cdot \mathscr{M}$

$$
=\left[\sum_{j=1}^{l} \frac{\partial N}{\partial \xi_{j}} \frac{\partial A}{\partial x_{j}}-\sum_{j=1}^{l} \frac{\partial D}{\partial \xi_{j}} \frac{\partial N}{\partial x_{j}}-\frac{\partial N}{\partial t}+N B\right] \cdot M
$$

where $M=M(x, t ; \xi)$ is the inverse matrix of $N(x, t ; \xi)$ and $\mathscr{M}=\mathscr{M}(x, t ; D)$ is a p.d.op. with the symbol $M(x, t ; \xi)$.

Let $R_{i}(x, t ; \xi)$ (resp. $L_{i}(x, t ; \xi)$ ) be an eigenvector of $A(x, t ; \xi)$ (resp. ${ }^{t} A(x, t ; \xi)$ ) corresponding to $\lambda_{i}(x, t ; \xi)$, then from the structures of $D, N$ and $M$ we have

Proposition 3.1. Condition (C.A) is equivalent to the following condition (C.B) :

$$
C_{i}(x, t ; \xi)=\left\langle L_{i},\left(\frac{\partial}{\partial t}-\sum_{j=1}^{l} A_{j} \frac{\partial}{\partial x_{j}}-B\right) R_{i}\right\rangle+\sum_{j=1}^{l} \frac{\partial \lambda_{i}}{\partial x_{j}}\left\langle L_{i}, \frac{\partial}{\partial \xi_{j}} R_{i}\right\rangle
$$

is identically zero for $(x, t ; \xi) \in \Omega \times R_{\xi}^{l},(i=1,2, \cdots, s)$.

We note that the condition (C.B) is independent of the choice of eigenvectors $R_{i}(x, t ; \xi)$ and $L_{i}(x, t ; \xi)$. Moreover we have

Proposition 3.2. The condition (C.B) is invariant under any space-like transformation.

From this proposition and the energy inequality, we have

Theorem 3.1. The solution of the Cauchy problem has finite propagation speed. More precisely its speed does not exceed $\lambda_{\max }$, where $\lambda_{\max }=\sup _{\substack{(x, t) \in \Omega,|\xi|=1 \\ i=1,2, \cdots, m-s}}\left|\lambda_{i}(x, t ; \xi)\right|$.

§4. Sufficiency of the condition (C.A). From the fact that the influence domain is finite we can deform the coefficients $A_{j}(x, t)$ in such a way that they are remain constant outside a small domain. This implies that the p.d.op. $N(x, t ; D)$ stated in Proposition 2.1 is invertible in the space $\mathscr{D}_{L^{2}}^{k}$. Next we deform $B(x, t)$ as a p.d.op. in such a way that the condition (C.A) is still valid for this system.

Now, the existence theorem is almost clear. Because, to solve (2.3) is now equivalent to solving (2.4) with $v=\Re u$. Next, this is equivalent further to solving (2.6) with $w=g v$. Notice also that $\mathscr{C} C u=g C M^{-1} \Re u$ $=g C \eta^{-1} g^{-1} w$ and $g C \eta^{-1} g^{-1}$ is of order 0 . Finally (2.6) is diagonalizable. Thus we have

Theorem 4.1. Suppose Condition (C.A), then for the given initial data $u_{0}(x) \in \mathscr{D}_{L^{2}}^{k}$ and any right-hand side $f(x, t) \in \mathcal{E}_{t}^{0}\left(\mathscr{D}_{L^{2}}^{k}\right)$ there exists a unique solution $u(x, t)$ of $(1.1)$ belonging to $\mathcal{E}_{t}^{0}\left(\mathscr{D}_{L^{2}}^{k-1}\right)$ and it satisfies the inequality (2.5).

$\S 5$. Necessity of the condition (C.A). Next we shall show the reciprocal statement of Theorem 4.1, namely we have

Theorem 5.1. Condition (C.A) is necessary for the uniformly $\mathcal{E}$ well-posedness of the Cauchy problem (1.1). 
For the proof of this theorem we use the method of energy estimates employed [2] and [4]; We suppose that the Cauchy problem is $\mathcal{E}$-well-posed and that at least one $C_{i}(x, t ; \xi)$ in $(3.2)$ is not identically zero. Then we can show that these two hypotheses induce a contradiction.

Acknowledgement. The author wishes to express his sincere gratitude to Professor S. Mizohata for his valuable advice.

\section{References}

[1] S. Mizohata: Systèmes hyperboliques. J. Math. Soc. Japan, 11, 205-233 (1959).

[2] - Some remarks on the Cauchy problem. J. Math. Kyoto Univ., 1, 109-127 (1961-1962).

[3] S. Mizohata, and Y. Ohya: Sur la condition de E. E. Levi concernant des équations hyperboliques. Publ. R. I. M. S., Kyoto Univ. Ser. A., 4, 511-526 (1968).

[4] — : Sur la condition d'hyperbolicité pour les équations a caractéristiques multiples. II. Jap. J. Math., 40, 63-104 (1971).

[5] L. Schwartz: Théorie des distributions. Hermann, Paris (1966).

[6] V. M. Petkov: On the Cauchy problem for first order hyperbolic systems with multiple characteristics. Soviet Math. Dokl., 14(2), 534-537 (1974).

[7] —-: Le problème de Cauchy et la propagation des singularités pour une class des systèmes hyperboliques non symétrisable. Ecol poly. Math. Semi. G-L-S. (1974-1975). 